\title{
Editorial Introduction
}

\author{
James P. Lynch · Alex R. Piquero
}

Published online: 18 February 2009

(C) Springer Science+Business Media, LLC 2009

It is an honor for us to be chosen as co-editors of the Journal of Quantitative Criminology. When Jamie Fox began the Journal it was innovative but served a very distinct readership - the small community of criminologists with a quantitative bent. This community has grown and, as a result, the prominence of the Journal has increased and stands among the most pre-eminent in criminology/criminal justice. The success of the Journal, however, is due not only to changes in the discipline but also to the careful stewardship of subsequent editors John Laub, Michael Maltz and David McDowall. They understood that the success of the Journal depended upon performing the mundane tasks of the review process promptly as well as adapting to broader changes in the discipline. They also understood the importance of working closely with the publisher to produce a quality product. We aspire to continue this tradition.

The mission of the Journal has evolved. Initially it was seen as an outlet for quantitatively oriented work in criminology that, at the time, could not find a suitable publication outlet. Now the amount and quality of quantitatively oriented criminology has increased and is broadly accepted in all of the discipline's journals. The danger in this evolution is that $J Q C$ may be regarded as only appropriate for even more esoteric methodological and statistical papers. This is not the case. $J Q C$ will retain its mission of publishing high quality, quantitatively-oriented criminology. This includes any paper of theoretical relevance to the field of criminology and criminal justice that employs quantitative methodology. The Journal will also be home to purely methodological pieces as long as the methodological issue being addressed can be shown to be relevant to work in criminology and criminal justice. This can include papers on research design, sampling, statistics, and measurement.

We are particularly interested in having $J Q C$ play a role in cross fertilization between criminology and other disciplines with more advanced quantitative methodologies. This is

J. P. Lynch (ه)

John Jay College of Criminal Justice, New York, NY, USA

e-mail: jlynch@jjay.cuny.edu

A. R. Piquero

University of Maryland, College Park, MD, USA

e-mail: apiquero@crim.umd.edu 
best done not by simply publishing work from these other disciplines, but by finding work that clearly states how these methods are relevant to issues of import in criminology and criminal justice. These methodologies must also be presented in a manner that is comprehensible to the majority of quantitatively-oriented criminologists. In short, we will strive to continue to make $J Q C$ among the field's leading journals in not only publishing the best quantitatively-oriented criminology and criminal justice research, but also in expanding the reach and applicability of this research to as wide an audience as possible. We invite your feedback and suggestions. 